\title{
Coherent X-ray diffraction imaging to investigate structure and morphological evolution of calcium carbonate microparticles
}

\author{
D. Younas ${ }^{1}$, F. K. Mürer ${ }^{1}$, S. Ucar ${ }^{2}$, Y. Chushkin ${ }^{3}$, F. Zontone ${ }^{3}$, \\ A. Gibaud ${ }^{4}$, D. W. Breiby ${ }^{1}$, B. Chattopadhyay ${ }^{1}$ \\ ${ }^{1}$ PoreLab, Department of Physics, Norwegian University of Science and Technology (NTNU), Trondheim, Norway \\ ${ }^{2}$ Department of Chemical Engineering, Norwegian University of Science and Technology, Trondheim, Norway \\ ${ }^{3} E S R F$, The European Synchrotron, Grenoble, France \\ ${ }^{4}$ LUNAM, IMMM, UMR 6283 CNRS, Faculté des Sciences, Le Mans Université, 72085 Le Mans, France \\ daniyal.younas@ntnu.no
}

Coherent X-ray diffraction imaging (CXDI) is a powerful lensless imaging technique utilizing X-rays with a high degree of coherence [1]. Conceptually, CXDI can image isolated microscopic objects with high resolution and since its first demonstration in 1999 [2] it has laid the foundation for the development of other methodologies such as ptychography and Bragg CXDI [1]. With the new lowemittance storage ring [3] at ESRF combined with a state-of-the-art Eiger 4M detector and efficient iterative algorithms, the ID10 beamline is optimised for CXDI experiments. Three-dimensional imaging of crystalline and amorphous particles at $\sim 14 \mathrm{~nm}$ resolution [4] has recently been demonstrated. In contrast to conventional characterisation methodologies such as electron microscopies, CXDI is ideally suited to study the surface morphology and interior of such microscopic particles without sectioning or ion-milling.

We used CXDI to image in 3D a series of $\mathrm{CaCO}_{3}$ microparticles prepared under different crystallization and growth conditions, revealing that the microparticles systematically assume a wide range of morphologies as function of temperature [5]. In this presentation, we demonstrate 3D CXDI imaging of $\mathrm{CaCO}_{3}$ particles 3-6 $\mu \mathrm{m}$ in diameter, with $16 \mathrm{~nm}$ voxel size. Wide-angle X-ray diffraction (WAXD) patterns [6] were recorded in combination with the CXDI datasets to identify the crystalline phase of the $\mathrm{CaCO}_{3}$ microparticles and obtain information of characteristic crystal planes. Figure 1 shows the evolution of $\mathrm{CaCO}_{3}$ particle morphology as a function of precipitation temperature: from the nested hexagonal morphology at $T=25^{\circ} \mathrm{C}$ to an appearance of spikes at $T=35^{\circ}$, and finally transforming to an extended rod-shape morphology at $T=45^{\circ} \mathrm{C}$. In addition, the internal structures of the particles and density variations within the particles can also be appreciated in 3D. Finally, we discuss the challenges arising due to radiation damage and how to resolve them, and also the future prospects of dynamic or serial CXDI experiments.

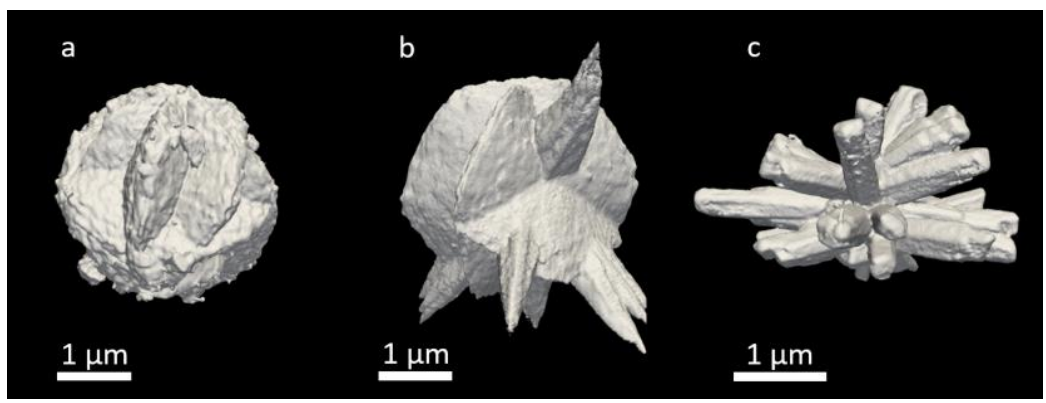

Figure 1. 3D iso-surface renderings of calcium carbonate particles precipitated in solution at a) $\left.25^{\circ} \mathrm{C} \mathrm{b}\right) 35^{\circ} \mathrm{C}$ c) $45^{\circ} \mathrm{C}$

[1] Chapman, H. N. \& Nugent, K. A. (2010). Nat. Photon. 4, 833.

[2] Miao, J., Charalambous, P., Kirz, J. \& Sayre, D. (1999). Nature 400, 342.

[3] ESRF News. (2017). No. 77, December 2017.

[4] Cherkas, O., Beuvier, T., Breiby, D. W., Chuskhin, Y., Zontone, F. \& Gibaud, A. (2017). Cryst. Growth Des. $17,4183$.

[5] Oral, M. Ç. \& Ercan, B. (2018). Powder Technology. 339, 781.

[6] Chushkin, Y., Zontone, F., Cherkas, O. \& Gibaud, A. (2019). J. Appl. Cryst. 52, 571.

Keywords: Coherent diffraction imaging; Calcium carbonate; 3D morphology

The authors thank the Research Council of Norway for funding through its Centre of Excellence program (No. 262644) and FRINATEK (No. 303252 and No. 275182).

Acta Cryst. (2021), A77, C918 